Proyecciones Journal of Mathematics

Vol. 28, No 2, pp. 133-139, August 2009.

Universidad Católica del Norte

Antofagasta - Chile

\title{
SCHUR RING AND QUASI-SIMPLE MODULES
}

\author{
PEDRO DOMÍNGUEZ-WADE \\ UNIVERSIDAD DE MATANZAS, CUBA \\ Received : November 2008. Accepted: April 2009
}

\begin{abstract}
Let $R$ be a ring of algebraic integers of an algebraic number field $F$ and let $G \leq G L_{n}(R)$ be a finite group. In this paper we show that the $R$-span of $G$ is just the matrix ring $M_{n}(R)$ of the $n \times n$-matrices over $R$ if and only if $G / O_{p_{i}}(G)$ is absolutely simple for all $p_{i} \in \pi$, where $\pi$ is the set of the positive prime divisors of $|G|$ and $O_{p_{i}}(G)$ is the largest normal $p_{i}$-subgroup.
\end{abstract}

Subjclass : Primary 20C20 ; Secondary 19A22.

Keywords : Schur ring, $\pi$-globally simple. 


\section{Introduction.}

Let $F$ be an algebraic number field with ring of algebraic integers $R$ and let $\pi=\left\{p_{1}, \ldots, p_{t}\right\}$ be a set of positive prime numbers. Assume that the $I_{i}$ are maximal ideals of $R$ such that $p_{i} \in I_{i}(i=1, \ldots, t)$. Set $L_{\pi}=\left\{\frac{a}{b} \mid\right.$ $\left.a, b \in R, b \notin I_{i}, i=1, \cdots, t\right\}$. Then $R_{\pi}$ denotes a localization of $R$ at $L_{\pi}$. Thus $R_{\pi}$ is a principal ideal having quotient field of characteristic zero and containing a unique prime ideal $I_{i}$ such that $p_{i} \in I_{i}, i=1, \ldots, n$. We denote the Jacobson radical of $R_{\pi}$ be $J\left(R_{\pi}\right)$. Therefore the residue ring $K_{m}=R_{\pi} / J\left(R_{\pi}\right)$ is a semi-simple ring of characteristic $m$. We may write

$$
K_{m}=\bigoplus_{i=1}^{t} k_{i}
$$

where the $k_{i}$ are fields of characteristic $p_{i}(i=1, \ldots, t)$.

If $G$ is a finite group then we obtain

$$
K_{m} G=\bigoplus_{i=1}^{t} k_{i} G .
$$

by (1.1).

From (1.2) it follows that

$$
1=f_{1}+\cdots+f_{t}
$$

where the $f_{i}$ are orthogonal central idempotents in $K_{m} G$.

Therefore

$\mathrm{K}_{m} G=\bigoplus_{i=1}^{t} k_{i} G$

$=\bigoplus_{i=1}^{t} K_{m} G f_{i}$ with $k_{i} G=K_{m} G f_{i}$.

Now, $R_{\pi}$ is a Hausdorff space in its $J\left(R_{\pi}\right)$-topology, i. e., that

$$
\bigcap_{j=1}^{\infty} J\left(R_{\pi}\right)^{j}=(0)
$$

Therefore the $J\left(R_{\pi}\right)$-adic completion $\hat{R}_{\pi}$ of $R_{\pi}$ is a complete semi-local ring such that

$$
K_{m}=R_{\pi} / J\left(R_{\pi}\right)=\hat{R}_{\pi} / J\left(\hat{R}_{\pi}\right)
$$

and

$$
\hat{R}_{\pi}=R_{1} \oplus \cdots \oplus R_{t}
$$


where the $R_{i}$ are complete local rings such that $R_{i} / J\left(R_{i}\right) \cong k_{i}$.

Observe that $R_{i}$ is isomorphic to $\hat{R}_{v_{p_{i}}}$, where $\hat{R}_{v_{p_{i}}}$ is a complete valuation ring corresponding to the discrete valuation $v_{p_{i}}$ associated to the maximal ideal $I_{i}$ of $R$. Thus, we may write

$$
\hat{R}_{\pi} \cong \hat{R}_{v_{p_{1}}} \oplus \cdots \oplus \hat{R}_{v_{p_{t}}}
$$

by (1.4).

Therefore

$$
\hat{R}_{\pi} G \cong \hat{R}_{v_{p_{1}}} G \oplus \cdots \oplus \hat{R}_{v_{p_{t}}} G .
$$

From ( 1.6) we obtain

$$
1=\hat{f}_{1}+\cdots+\hat{f}_{n}
$$

where the $\hat{f}_{i}$ are orthogonal central idempotents in $\hat{R}_{\pi} G$.

Let $\pi_{l}$ be any set of positive prime numbers. Assume that $R_{\pi_{l}}$ is the localization of $R$ at $L_{\pi_{l}}$. Then we have

$$
\bigcap_{l=1}^{\infty} R_{\pi_{l}}=R
$$

In the study of the Schur ring $M_{n}(R)$ of a commutative ring $R$ the main problem is to find a finite group $G \leq G L_{n}(R)$ such that the $R$-span of $G$ coincides with the matrix ring $M_{n}(R)$ of the $n \times n$-matrices on $K$. The more precise question, in general sense, which Azumaya algebras over $R$ are obtainable as an epimorphic image of the group-ring $R G$ for some finite group $G$.

\subsection{Notations and Definitions.}

Throughout the paper $F$ denote an algebraic number field and $R$ denote the ring of algebraic integers of $F$. Moreover, $K_{m}$ is semi-simple ring of characteristic $m$ with maximal ideals $K_{i}$ and residue fields $k_{i}=K_{m} / K_{i}$ of characteristic $p_{i}$. For an maximal ideal $I$ of $R$ we denote by $\phi$ the $I$-adic

valuation on $F$. Here $R_{v_{p}}$ denote the valuation ring of $v_{p}$ and $\hat{R}_{v_{p}}$ denote the complete valuation ring corresponding to the discrete valuation $v_{p}$. Let $M_{n}(R)$ stand for the ring of $(n \times n)$-matrices over $R$. We write $G L_{n}(R)$ for the multiplicative group of the invertible elements of $M_{n}(R)$. For a finite subgroup $G$ of $G L_{n}(R)$ we let $\langle G\rangle_{R}$ be the $R$-span of $G$ in $M_{n}(R)$. Let $\pi$ be a set of natural primes. We denote the fields of rational and complex numbers by $\mathbf{Q}$ and $\mathbf{C}$, respectively. 


\section{Preliminary Results.}

Let $R$ be a commutative ring and let $G \leq G L_{n}(R)$ be a finite group. Then the matrix ring $M_{n}(R)$ is called Schur ring if $\langle G\rangle_{R}=M_{n}(R)$.

Lemma 2.0.1. Let $k$ be a field of characteristic $p$ and let $G \leq G L_{n}(k)$ be a finite group. Assume that $V$ is the $k G$-module corresponding to $G$. Then $G$ is absolutely simple if and only if $\langle G\rangle_{k}=M_{n}(k)$.

Proof. If $G$ is absolutely simple then by Burnside's theorem the assertion follows. Conversely, let $\dot{G}$ be a finite group with a representation $\varphi: \dot{G} \longrightarrow G L_{n}(k)$ such that $\varphi(\dot{G})=G$. Consider the subjection of $k$-algebras $\psi: k \dot{G} \longrightarrow\langle G\rangle_{k}$, where $\psi(\dot{G})=\varphi(\dot{G})$. Therefore $k \dot{G} / \operatorname{ker} \psi \cong M_{n}(k)$. Since $J(k \dot{G}) \subseteq \operatorname{ker} \psi$ if follows that the matrix algebra summand of $k \dot{G} / J(k \dot{G})$ corresponding to $V$ is $M_{n}(k)$, so the result follows.

Let $K_{m}$ be a semi-simple ring of characteristic $m$ and let $G \leq G L_{n}\left(K_{m}\right)$ be a finite group. Assume that $V$ is the $K_{m} G$-module corresponding to $G$. Then $G$ is called $\pi$-quasi-simple if each direct summand $V f_{i}$ is an absolutely simple $k_{i} G$-module.

Lemma 2.0.2. Let $K_{m}$ be a semi-simple ring of characteristic $m$ and let $G \leq G L_{n}\left(K_{m}\right)$ be a finite group. Assume that $V$ is the $K_{m} G$-module corresponding to $G$. Then $\langle G\rangle_{K_{m}}=M_{n}\left(K_{m}\right)$ if and only if $G$ is $\pi$-quasisimple.

Proof. We have

$$
\begin{aligned}
& \langle G\rangle_{K_{m}}=\langle G\rangle_{k_{1}} \oplus \cdots \oplus\langle G\rangle_{k_{t}} \\
= & M_{n}\left(K_{m}\right) \\
= & M_{n}\left(k_{1}\right) \oplus \cdots \oplus M_{n}\left(k_{t}\right)
\end{aligned}
$$

Therefore $\langle G\rangle_{k_{i}}=M_{n}\left(k_{i}\right)$. Thus applying the last lemma the result follows. Conversely, applying again the lemma (2.0.1) we deduce that

$$
\langle G\rangle_{k_{i}}=M_{n}\left(k_{i}\right)
$$

for all $i$. Thus we may write

$$
\begin{array}{rcc}
\langle G\rangle_{K_{m}} & = & \langle G\rangle_{k_{1}} \oplus \cdots \oplus\langle G\rangle_{k_{t}} \\
& = & M_{n}\left(k_{1}\right) \oplus \cdots \oplus M_{n}\left(k_{t}\right) \\
& = & M_{n}\left(K_{m}\right)
\end{array}
$$


So we are done.

Proposition 2.0.3. Let $G \leq G L_{n}(\mathbf{C})$ be a finite group. Assume that $F$ is a finite extension of $\mathbf{Q}$ such that $G \leq G L_{n}(F)$. If $R$ is the ring of integers of $F$ then $G \leq G L_{n}\left(R_{\pi}\right)$, being $R_{\pi}$ a localization of $R$ at $L_{\pi}$.

Proof. Let $\hat{R}_{\pi}$ be the completion of $R_{\pi}$. It is well known that $G \leq$ $G L_{n}\left(\hat{R}_{v_{p_{l}}}\right)(l=1, \ldots, t)$, where $\hat{R}_{v_{p_{l}}}$ is a complete valuation ring. Let $\hat{U}_{i}$ be the $\hat{R}_{v_{p_{l}}} G$-module corresponding to $G$. From (1.5) we deduce that there is an $\hat{R}_{\pi} G$-module minimal $\hat{R}_{\pi}$-free $\hat{U}=\hat{U}_{1} \oplus \cdots \oplus \hat{U}_{t}$, so $G \leq G L_{n}\left(\hat{R}_{\pi}\right)$. Since $\hat{U}$ is a complete minimal $\hat{R}_{\pi^{-}}$free module it follows that $\hat{U}=\hat{R}_{\pi} U$, being $U$ an $R_{\pi} G$-module, which is a Hausdorff space for its $J\left(R_{\pi}\right)$-topology. So we are done.

From (1.8) and the last proposition one can deduce an "Hasse Principle" for $\langle G\rangle_{R}$ to coincide with $M_{n}(R)$ : this is the case if and only if $\langle G\rangle_{R_{\pi}}=$ $M_{n}\left(R_{\pi}\right)$ for every set of positive prime numbers $\pi$.

Let $G \leq G L_{n}(\mathbf{C})$ be a finite group and let $U_{\mathcal{C}}$ be the $\mathbf{C} G$-module corresponding to $G$. Then there exists a finite extension $F$ of $\mathbf{Q}$ with $F G$ module $U_{F}$ conjugate to $U_{\mathcal{C}}$. According to the proposition (2.0.3) there is an $R_{\pi} G$-module $U$ which is also conjugate to $U_{\mathcal{C}}$. Let $\hat{R}_{\pi}$ be the $J\left(R_{\pi}\right)$-adic completion of $R_{\pi}$. We know that there exists an $\hat{R}_{\pi} G$-module $\hat{U}$ which is the completion of $U$. From (1.7) it follows that

$$
\hat{U}=\hat{U} \hat{f}_{1} \oplus \cdots \oplus \hat{U} \hat{f}_{t}
$$

where the $\hat{U} \hat{f}_{i}$ are $R_{v_{p_{i}}} G$-modules. The $K_{m} G$-module $\bar{U}_{K_{m}}=\hat{U} / J\left(R_{\pi}\right) \hat{U}$ $=\hat{U} \hat{f}_{1} / J\left(R_{v_{p_{1}}}\right) \hat{U} \hat{f}_{1} \oplus \cdots \oplus \hat{U} \hat{f}_{t} / J\left(R_{v_{p_{t}}}\right) \hat{U} \hat{f}_{t}$

is called reduction of $U$ modulo $\pi$.

The natural projection $G L_{n}\left(\hat{R}_{\pi}\right) \rightarrow G L_{n}\left(K_{m}\right)$ induces the homomorphism $\tau: G \rightarrow G L_{n}\left(K_{m}\right)$, where $\tau(G)=\bar{G}$. Furthermore the homomorphism $G L_{n}\left(\hat{R}_{v_{p_{i}}}\right) \rightarrow G L_{n}\left(k_{i}\right)$ induces the homomorphism $\tau_{i}: G \rightarrow G L_{n}\left(k_{i}\right)$ with $\tau_{i}(G)=\bar{G}_{i}$

Observe that if $\langle G\rangle_{R_{\pi_{l}}}=M_{n}\left(R_{\pi_{l}}\right)$, where $\pi_{l}$ is the set of the positive prime divisors of $|G|$, then $\langle G\rangle_{R_{\pi}}=M_{n}\left(R_{\pi}\right)$ for every set $\pi$ of positive prime numbers. We use Nakayama's lemma to show that that $\langle G\rangle_{R_{\pi_{l}}}=M_{n}\left(R_{\pi_{l}}\right)$ is equivalent to $\langle\bar{G}\rangle_{K_{m}}=M_{n}\left(K_{m}\right)$. 


\section{Main Results.}

Let $R$ be a ring of algebraic integers and let $G \leq G L_{n}(R)$ be a finite group. Assume that $\pi$ is the set of the positive prime divisors of $|G|$ and that $U$ is the $R_{\pi} G$-module corresponding to $G$. If the reduction of $U$ module $\pi$ is a $\pi$-quasi-simple $K_{m} G$-module, then we say that $G$ is a $\pi$-globally simple.

Lemma 3.0.4. Let $R$ be a ring of algebraic integers with localization $R_{\pi}$ and let $G \leq G L_{n}\left(R_{\pi}\right)$ be a finite group. Assume that $\pi$ is a set of the positive prime divisors of $|G|$. Then $\langle G\rangle_{R_{\pi}}=M_{n}\left(R_{\pi}\right)$ if and only if $G$ is $\pi$-globally simple.

Proof. From $\langle G\rangle_{R_{\pi}}=M_{n}\left(R_{\pi}\right)$ we deduce that $\langle\bar{G}\rangle_{K_{m}}=M_{n}\left(K_{m}\right)$, being $K_{m}=R_{\pi} / J\left(R_{\pi}\right)$ a semi-simple ring. The result follows by lemma (2.0.2). Conversely, applying again the lemma $(2.0 .2)$ we obtain $\langle\bar{G}\rangle_{K_{m}}=M_{n}\left(K_{m}\right)$, so the assertion follows.

Theorem 3.0.5. Let $R$ be a ring of algebraic integers and let $G \leq G L_{n}(R)$ be a finite group. Then $\langle G\rangle_{R}=M_{n}(R)$ if and only if $G$ is $\pi$-globally simple.

Proof. From $\langle G\rangle_{R}=M_{n}(R)$ we deduce that $\langle G\rangle_{R_{\pi}}=M_{n}\left(R_{\pi}\right)$. Hence we obtain $\langle\bar{G}\rangle_{K_{m}}=M_{n}\left(K_{m}\right)$. The result follows by lemma (2.0.2). Conversely, since

$$
\langle G\rangle_{R_{\pi}}=M_{n}\left(R_{\pi}\right)
$$

by lemma (3.0.4) we deduce that for any set of prime numbers $\pi$ we obtain

$$
\langle G\rangle_{R_{\pi}}=M_{n}\left(R_{\pi}\right)
$$

So we are done.

Theorem 3.0.6. Let $G \leq G L_{n}(\mathbf{C})$ be a finite group. Then $G$ is $\pi$-globally simple if and only if $G / O_{p_{i}}(G)$ is absolutely simple.

Proof. Let $F$ be a finite extension of $\mathbf{Q}$ such that $G \leq G L_{n}(F)$, and let $R$ be the ring of integers of $F$. Consider the homomorphism $h_{i}: G \rightarrow \bar{G}_{i}$. Since $\bar{G}_{i}$ is absolutely simple it follows that $\operatorname{ker} h_{i}=O_{p_{i}}(G)$. Conversely, since $G / O_{p_{i}}(G)$ is absolutely simple for all $i$, we deduce that $G$ is $\pi$-quasisimple, being $\pi$ the set of positive prime divisors of the order of $G$. So we are done. 


\title{
References
}

[1] P. HuU Tiep. Globally irreducible representations of the finite symplectic group Sp4(q), Comm. in Algebra 22, pp. 6439 - 6457, (1994).

[2] P. HuU TieP. Basic spin representations of 2Sn and 2An as globally irreducible representations, Archiv Math. 64, pp. 103 - 112, (1995).

[3] P. HuU TiEP. Weil representations as globally irreducible representations, Math. Nachr. 184, pp. 313 - 327, (1997).

[4] P. HuU TieP. Globally irreducible representations of finite groups and integral lattices, Geomet. Dedicata 64, pp. 85 - 123, (1997).

[5] P. Spiga And Q. Wang An answer to Hirasaka and Muzychuk : Every $p$-Schurring over $C_{p}^{3} \mathrm{p}$ is Schurian, Discrete Mathematics 308, pp. 1760-1763, (2008).

[6] A. E. Zalesskit and F.Van Oystaeyen Finite Groups over Arithmetical Rings and Globally Irreducible Representations, J. Algebra 215, pp. 418-436, (1999).

\author{
Pedro Domínguez Wade \\ Department of Mathematics \\ Matanzas University \\ Carretera Matanzas \\ Varadero Km. 3 \\ Cuba \\ e-mail : pedro.dominguez@umcc.cu
}

